\title{
Spatiotemporal changes of cold surges in Inner Mongolia between 1960 and 2012
}

\author{
LIU Xianfeng ${ }^{1,2},{ }^{*}$ ZHU Xiufang ${ }^{1,2}$, PAN Yaozhong ${ }^{1,2}$, ZHAO Anzhou $^{1,2}$, \\ LI Yizhan ${ }^{1,2}$ \\ 1. State Key Laboratory of Earth Surface Processes and Resource Ecology, Beijing Normal University, Beijing \\ 100875, China; \\ 2. College of Resources Science \& Technology, Beijing Normal University, Beijing 100875, China
}

\begin{abstract}
In this study, we analyzed the spatiotemporal variation of cold surges in Inner Mongolia between 1960 and 2012 and their possible driving factors using daily minimum temperature data from 121 meteorological stations in Inner Mongolia and the surrounding areas. These data were analyzed utilizing a piecewise regression model, a Sen+MannKendall model, and a correlation analysis. Results demonstrated that (1) the frequency of single-station cold surges decreased in Inner Mongolia during the study period, with a linear tendency of -0.5 times/10a ( -2.4 to 1.2 times/10a). Prior to 1991 , a significant decreasing trend of -1.1 times/10a ( -3.3 to 2.5 times/10a) was detected, while an increasing trend of 0.45 times/10a ( -4.4 to 4.2 times/10a) was found after 1991 . On a seasonal scale, the trend in spring cold surges was consistent with annual values, and the most obvious change in cold surges occurred during spring. Monthly cold surge frequency displayed a bimodal structure, and November witnessed the highest incidence of cold surge. (2) Spatially, the high incidence of cold surge is mainly observed in the northern and central parts of Inner Mongolia, with a higher occurrence observed in the northern than in the central part. Inter-decadal characteristic also revealed that high frequency and low frequency regions presented decreasing and increasing trends, respectively, between 1960 and 1990. High frequency regions expanded after the 1990s, and regions exhibiting high cold surge frequency were mainly distributed in Tulihe, Xiao'ergou, and Xi Ujimqin Banner. (3) On an annual scale, the cold surge was dominated by AO, NAO, CA, APVII, and CQ. However, seasonal differences in the driving forces of cold surges were detected. Winter cold surges were significantly correlated with $A O$, NAO, SHI, CA, TPI, APVII, CW, and IZ, indicating they were caused by multiple factors. Autumn cold surges were mainly affected by $C A$ and $I M$, while spring cold surges were significantly correlated with CA and APVII.
\end{abstract}

Keywords: cold surge; piecewise regression; Sen + Mann-Kendall; spatiotemporal variations; influencing factors; Inner Mongolia

Received: 2014-09-25 Accepted: 2014-10-31

Foundation: Major Project of High-resolution Earth Observation System

Author: Liu Xianfeng (1986-), PhD Candidate, specialized in resource and environmental remote sensing and disaster remote sensing. E-mail: liuxianfeng7987@163.com

*Corresponding author: Zhu Xiufang (1982-), PhD and Associate Professor, specialized in agricultural and disaster monitoring, and interaction between land use/cover and climate change. E-mail: zhuxiufang@bnu.edu.cn 


\section{Introduction}

Extreme climatic events are defined as significant statistical deviations of certain climatic factors that meet or exceed specific upper and lower limit threshold observed or statistical values (Zheng et al., 2014). Compared to the climate mean state, extreme climatic events are often unusual, sudden, and unpredictable. As extreme climatic events are highly sensitive to climate change (Cui et al., 2009), they pose a large and far-reaching threat to human society and the natural environment (Easterling et al., 2000). According to the Fifth IPCC Report, global mean temperature increased by $0.72{ }^{\circ} \mathrm{C}\left(0.49-0.89{ }^{\circ} \mathrm{C}\right)$ between 1951 and 2012 (Stocker et al., 2013), and the mean land surface temperature in China increased by $1.38^{\circ} \mathrm{C}$ from 1960 to 2009. The growth rate of the mean land surface temperature in China was much higher than observed globally or for the Northern Hemisphere over the same period (NARCCC, 2011). The most obvious warming occurred in northern China, which triggered an increase in the frequency and intensity of extreme climatic events (Zhai et al., 1997; Zhai et al., 1999). Due to the worsening of extreme climatic events such as floods, droughts, heat waves, and cold surges, global climate change has received attention by both scholars and the public as a scientific issue of major concern. Cold surges, which represent the primary low-temperature extreme weather event, most directly impact and threaten socio-economic health, ecological systems, and human health. Therefore, cold surges have become a primary focus of scientific researchers.

With the increasing concern over global climate change, research of extreme climatic events such as cold surges has become the core domestic and international research issue related to comprehensive prevention and disaster mitigation (Easterling et al., 2000). As the primary winter-month disaster, cold surges have caused serious socio-economic losses. In 1955, Mr. Li Xianzhi studied the outbreak of the East Asian cold surge process and divided the cold surge into three types (Li et al., 1955). Subsequently, Mr. Tao Shiyan analyzed the cold air source and path of cold surges influencing China (Tao et al., 1957). In addition, Wang Zunya (Wang et al., 2006) and Qian Weihong (Qian et al., 2007) analyzed the spatiotemporal variation of the cold surge and its influence on China, respectively. The former suggested that the decrease in cold surge frequency could be attributed to the weakening of the Siberian high and the winter monsoon. Comparatively, the latter pointed out that the decreasing frequency of cold surges, cool events, and high winds collectively induced the increase of average minimum winter temperatures and the formation of continuous warm winters. In reality, the trend of decreased cold surges was not consistent across all regions; significant heterogeneity was observed among regions, and some regions even experienced an increase in cold surges (Zhou et al., 2011; Chen et al., 2008; Wang et al., 2010; Mansur et al., 2012). Under the influence of global warming, the vulnerability of ecological systems to extreme cold events gradually increased. Therefore, the understanding of the spatiotemporal variation of cold surges plays a key role in the sustainable development of ecosystem health.

Most of Inner Mongolia is covered by grasslands, and these grasslands account for $20 \%$ of the area of all natural grasslands in China (Guo et al., 2013). Unfortunately, grasslands are highly sensitive to global climate change, and Inner Mongolia represents one of the most severely affected regions by cold surges in China. The increase of extreme climatic events raised the uncertainty associated with meteorological disasters, which in turn increased the 
risks to agricultural production and endangered ecological and food security (Wang et al., 2013). The recent spatiotemporal variation of cold surges in Inner Mongolia and their possible driving forces remains unclear. Thus, in order to provide support for comprehensive disaster prevention and mitigation, this paper analyzed the spatiotemporal variation of cold surges in Inner Mongolia and their possible influencing factors from a geographic perspective between 1960 and 2012. The variations were modeled using a piecewise regression model, a Sen + Mann-Kendall model, and correlation analysis, and daily minimum temperature data from 121 meteorological stations in Inner Mongolia and the surrounding areas were utilized.

\section{Data and method}

\subsection{Study area}

Inner Mongolia, located in the northern frontier of China $\left(37^{\circ} 24^{\prime}-53^{\circ} 23^{\prime} \mathrm{N}\right.$ and $97^{\circ} 12^{\prime}-$ $126^{\circ} 04^{\prime} \mathrm{E}$ ), represents an important ecological barrier in northern China (Figure 1). Regional climate in this area is characterized by a transitional zone from the arid/semi-arid climate to the west to the humid/semi-humid climate associated with the monsoons of the southeastern coast (Sun et al., 2010), where the Da Hinggan and the Yinshan Mountains form an important natural boundary that induces differences in climate. From the northeast to the southwest, vegetation types are forests, grasslands and deserts. At the same time, as the major production base for animal husbandry in China, the ecological environment of Inner Mongolia has further deteriorated in recent decades from global climate warming and human activities. Furthermore, most of the vegetations are distributed in the arid and semi-arid farming-pastoral areas where the ecological environment is extremely fragile. Thus, Inner Mongolia represents one of the regions sensitive to global cli-

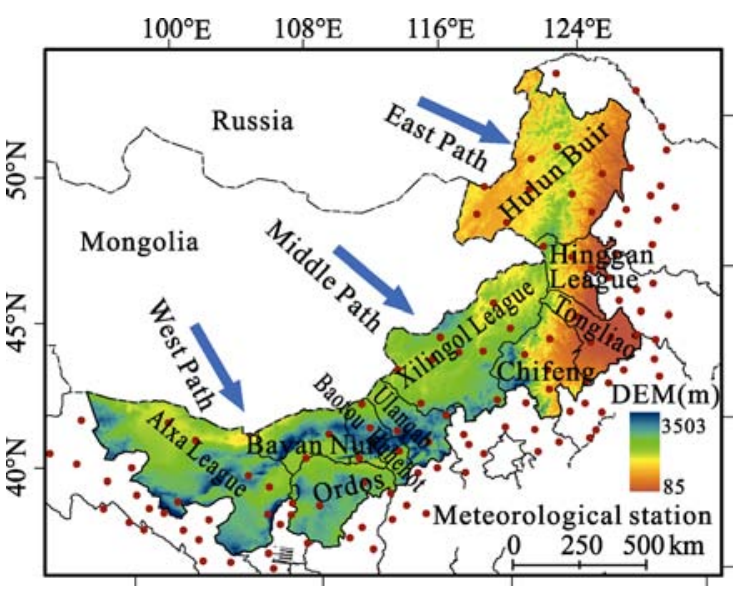

Figure 1 Study area and distribution of meteorological stations mate change.

\subsection{Data source and precipitation}

In order to ensure the integrity and continuity of meteorological datasets and to establish uniform and stable temperature sequences, this study utilized daily minimum temperature data from 121 meteorological stations within the study area and its peripheries between 1960 and 2013. Data was chosen considering continuity and the longest-period rules and was collected from the China Meteorological Data Sharing Service System (http://cdc.cma.gov.cn). To eliminate the impact of non-climatic factors, all data was selected only after a rigorous quality inspection and control, including extreme value tests, and a time consistency review. Missing data for some sites were interpolated from neighboring stations. It should be noted 
that the reason we selected the neighboring station interpolation method is that the linear regression interpolation method can bias cold surge frequency results by smoothing the temperature difference between two days. In addition, this paper defined cold surge frequency in a given year as the frequency of cold surges between September 1 of a given year and May 31 of the following year (i.e., the cold surge frequency in 1960 was calculated as the number of cold surges that occurred between September 1, 1960 and May 31, 1961).

In order to analyze factors influencing cold surge variability, this paper selected a series circulation factors that are highly correlated with regional temperature variability. The Siberian High Index (SHI) was calculated from monthly sea-level-pressure data obtained from NCEP/NCAR reanalysis data. The monthly cold air (CA), the Tibetan Plateau Index (TPI), the Asian polar vortex intensity index (APVII), the East Asian trough intensity (CQ), the East Asian trough position (CW), the Asian zonal circulation index (IZ), and the Asia meridional circulation index (IM) were derived from 74 circulation index data sets published by the national climate center on climate change and prediction research associated with the China Bureau of Meteorology. The Arctic Oscillation (AO) and the monthly North Atlantic Oscillation (NAO) were obtained from the climate prediction center associated with the National Oceanic and Atmospheric Administration (http://www.cpc.ncep.noaa.gov/).

In this study, the ecological zone boundary was collected from Terrestrial Ecoregions data published by the World Wildlife Fund (http://www.worldwildlife.org/). This dataset divided the globe into 825 ecological zones, of which 13 contained portions of Inner Mongolia $(\mathrm{Mu}$ et al., 2012). Inner Mongolia was divided into three primary ecological zones in accordance with climatic characteristics, vegetation distribution, and information from previous studies (John et al., 2008): the eastern forest ecological zone (Region I), the central grassland ecological zone (Region II), and the western desert ecological zone (Region III).

\subsection{Methods}

\subsubsection{Definition of cold surge}

According to the cold surge grade (GB/T21987-2008) and the cold air grade (GB/T204842006) as defined in the National Standards of the People's Republic of China, a single station cold surge is defined as a drop in the daily minimum or daily mean temperature of at least $8{ }^{\circ} \mathrm{C}$ over a period of 24 hours, at least $10^{\circ} \mathrm{C}$ over a period of 48 hours, or at least $12^{\circ} \mathrm{C}$ over a period of 72 hours, with an air minimum temperature of no greater than $4^{\circ} \mathrm{C}$. A regional cold surge is defined as a cold surge event, where more than $40 \%$ of the observation stations experienced a single station cold surge, as defined above. As the daily average temperature is calculated from the daily maximum and minimum temperatures, it does not represent the actual measured temperature. The minimum temperature is independent of solar radiation effects and, thus, may better reflect the relative magnitude of temperature drops (Qian et al., 2007). Therefore, in our paper, we calculated the frequency of cold surge based on the minimum temperature.

\subsubsection{Sen Trend analysis and Mann-Kendall statistical test}

The trend in the frequency of cold surges in Inner Mongolia was calculated using a Sen Trend analysis (Sen et al., 1968), and the significance of the trend was further examined by the Mann-Kendall statistical test (Kendall et al., 1948). The advantage of Sen Trend analysis 
is that this method does not require a specific sample distribution and is free from the inference of abnormal values. Therefore, this method is robust and resistant to errors from measurements or outlier data. The formula is as follows:

$$
\beta=\operatorname{mean}\left(\frac{x_{j}-x_{i}}{j-i}\right) \forall \mathrm{j}>i
$$

where $\beta$ represents the trend in cold surge frequency; $i$ and $j$ represent the sequence of years; and $x_{i}$ and $x_{j}$ denote the frequency of cold surge at time $i$ or $j$, respectively. When $\beta>0$, the frequency of cold surges displays an increasing trend, and when $\beta<0$, the frequency of cold surges displays a decreasing trend. The predicted trend is considered significant when the Mann-Kendall test indicates a significance level of at least 0.05 .

\subsubsection{Piecewise linear regression model}

To detect the consistency of a trend in the frequency of a cold surge in Inner Mongolia during the study period, we utilized a piecewise linear regression model. Because of its ability to detect a turning point in long time-series data and compensate for flaws that do not truly reflect trends detected by ordinary linear regression models, this method has been widely used in climate analyses (Tomé et al., 2004; Sun et al., 2011). The formulas are as follows:

$$
y= \begin{cases}\beta_{0}+\beta_{1} t+\varepsilon, & t \leqslant \alpha \\ \beta_{0}+\beta_{1} t+\beta_{2}(t-\alpha)+\varepsilon, & t>\alpha\end{cases}
$$

where $y$ is frequency of cold surge; $t$ is the year; $\alpha$ is the estimated turning point (TP) of the time-series; $\beta_{0}$ is the intercept; $\beta_{1}$ and $\beta_{1}+\beta_{2}$ denote the magnitude of the trends before and after the TP, respectively; and $\varepsilon$ is the residual random error. Least-squares linear regression was used to estimate other coefficients. A $p$ value of less than 0.05 was considered significant.

\section{Results}

With the increase of frequency and intensity of extreme event, the spatiotemporal variation of cold surges and its driving forces in Inner Mongolia need an overall analysis. In this section, we investigated the temporal variations of cold surges, spatial differential characteristics of cold surges and trend analysis of cold surges in Inner Mongolia between 1960 and 2012.

\subsection{Temporal variations of cold surges}

\subsubsection{Regional scale}

The frequency of occurrence of single-station cold surges in Inner Mongolia revealed a decreasing trend between 1960 and 2012, with a linear tendency of -0.5 times/10a $(p<0.01)$. Obvious stage characteristics in the frequency of cold surges are presented in Figure 2a. Based on a piecewise linear regression model, a turning point in the frequency of cold surges was detected in 1991, and we further fitted the linear tendencies of the two separated periods. We found that (1) a significant decreasing trend of -1.1 times/10a $(\mathrm{p}<0.01)$ was detected prior to 1991, while (2) an increasing trend of 0.45 times $/ 10 \mathrm{a}(\mathrm{p}=0.21$ ) was found after 1991. In addition, the variability of the frequency of two-day and three-day cold surges was 
determined, and results revealed that both types of cold surges experienced a turning point. However, differences in trends were also revealed. (1) A significant decreasing trend $(-0.016$ times/10a) in two-day cold surges was detected during the study period, of which an extremely significant decreasing trend was present before 1985 with no significant trend after 1985. (2) Three-day cold surges showed a slight decreasing trend $(-0.0008$ times/10a) during the study period, of which a significant decreasing trend ( -0.014 times/10a) was detected before 1984, and a significant increasing trend (0.01 times/10a) was found after 1984.

On a seasonal scale, cold surges in later spring posed serious threats to local agricultural production. Thus, we analyzed the trend of spring cold surges. We found that the trend in spring cold surges was consistent with that of the annual value, with a turning point in 1991 (Figure 2b). A decreasing trend of -0.5 times/10a $(\mathrm{p}<0.01)$ was detected before 1991, which was higher than -0.4 times/10a $(\mathrm{p}<0.01)$ determined for autumn and -0.2 times/10a $(\mathrm{p}<0.01)$ determined for winter. While a slight increasing trend was present after 1991, the sequence of linear tendency was spring $(0.165$ times/10a) $>$ autumn $(0.164$ times/10a) $>$ winter (0.122 times/10a). From the analysis above, we concluded that the most obvious change in cold surges occurred in spring, whereas the frequency was relative stable during winter.
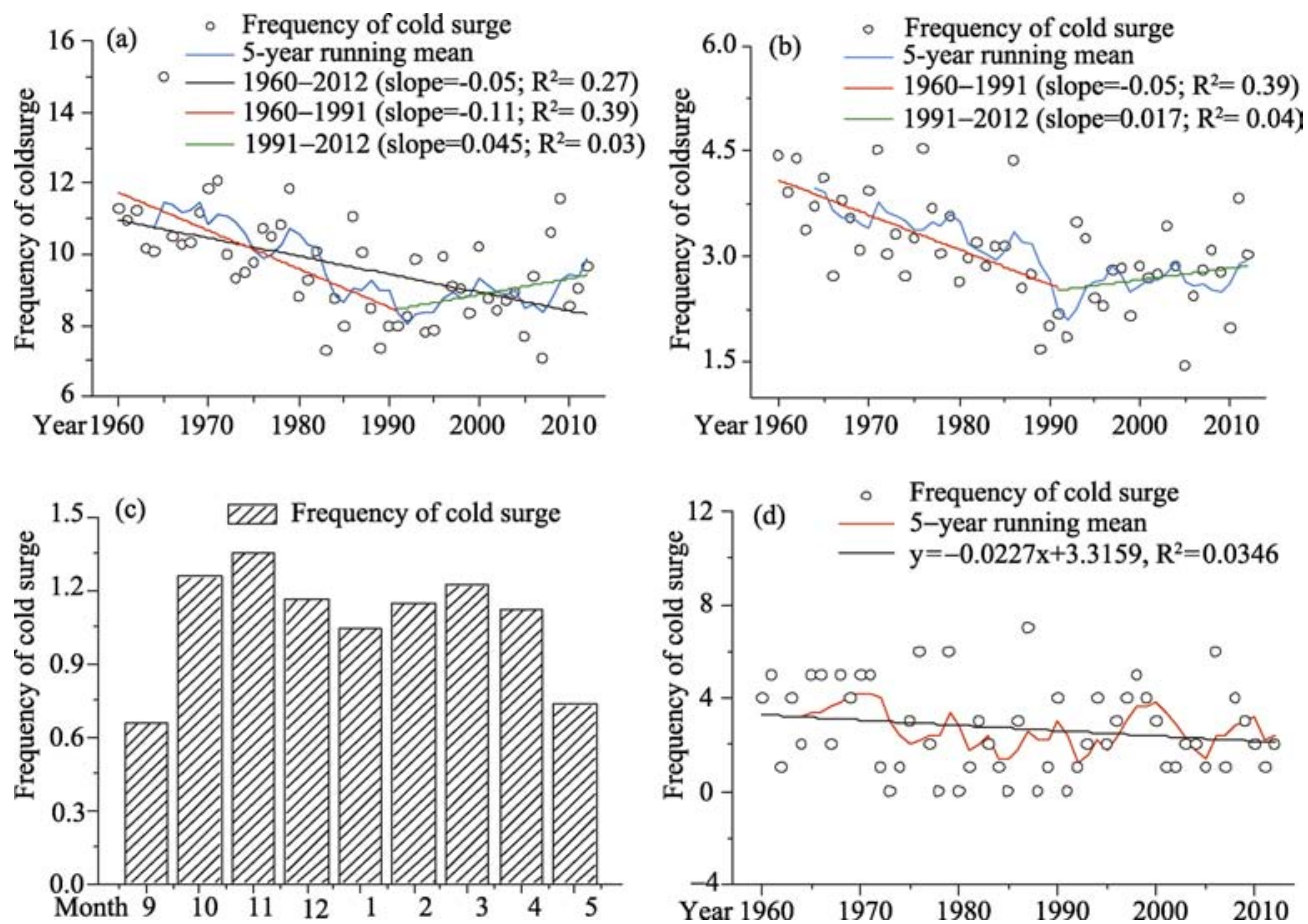

Figure 2 Temporal variation of cold surge frequency in Inner Mongolia from 1960 to 2012: a) The annual variation of cold surges at a single station; b) The variation of spring cold surges at a single station; c) Monthly variation of cold surges at a single station; d) Regional variation of cold surges

The frequency of monthly cold surges displayed a bimodal structure. The highest frequency of cold surges was during October, November, and March of the following year. Of these, November experienced the highest number of cold surges (1.36 times), followed by October and March with 1.26 and 1.22 cold surges, respectively. The lowest frequency oc- 
curred during September, with 0.66 cold surges (Figure 2c).

The regional cold surge occurred 147 times in Inner Mongolia between 1960 and 2012, with an average of 2.8 times per year. The earliest season cold surge occurred on September 9, 2006, while the latest occurred on May 13, 1976. On a decadal scale, the highest cold surge frequency occurred during the 1960s, with a total frequency of 37 times, followed by a frequency of 29 in the 1970s. The frequency of cold surges decreased further in the 1980s and 1990s to 18 occurrences, whereas the frequency between 2000 and 2012 rebounded back to 29 times. Annually, the frequency of the regional cold surge in Inner Mongolia exhibited a decreasing trend from 1960 to 2012, with a linear tendency of -0.23 times /10a (Figure 2d).

\subsubsection{Single station scale}

In order to understand the spatiotemporal variation of cold surges in Inner Mongolia, we further analyzed the annual variability of cold surges at each station from 1960 to 2012 (Figure 3). We found that cold surges occurred more frequently before 1980, especially in 1965 when high value was observed for almost all stations. Contrastingly, a relatively low cold surge frequency was detected from 1980 to 1995 followed by an increasing trend in cold surge frequency after 1995. Due to the increase in the frequency and intensity of extreme climate events during recent years, we further investigated the variability of the frequency of cold surges from 2000 to 2012. Results showed a decreasing trend in forested regions, with a linear tendency of -4.4 to 4.0 times/10a. A similar trend was detected for

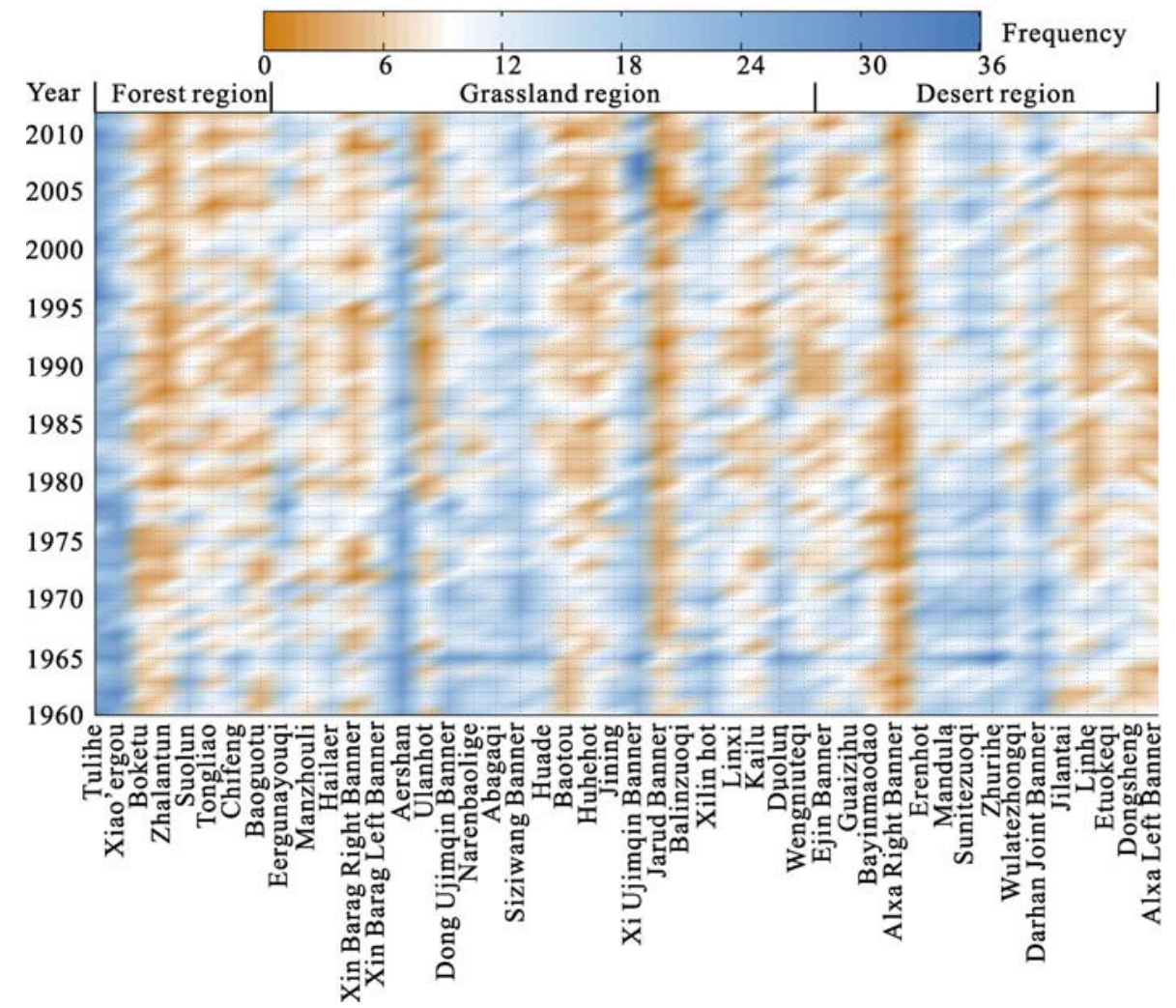

Figure 3 Annual variations of cold surges at each station in Inner Mongolia from 1960 to 2012 
grassland regions (-6.2 to 2.5 times/10a). However, more stations with extremely high values were contained within grassy regions, suggesting the intensity of extreme climatic events increased and, thus, ecosystem impacts increased. Compared to forested and grassy regions, the frequency of cold surges in desert regions presented an increasing trend ( -3.0 to 6.3 times/10a). On an annual scale, regions with more cold surge occurrences (blue band) were mainly distributed in Tulihe, Xiao'ergou, Aershan, Xi Ujimqin Banner, and Darhan Joint Banner. Of these, Tulihe experienced the highest cold surge frequency, while the frequency of cold surges in Xi Ujimqin Banner significantly increased in recent years.

\subsection{Spatial differential characteristics of cold surge}

\subsubsection{Spatial distribution of cold surges}

Significant spatial variations were observed for cold surges in Inner Mongolia. A high cold surge frequency was observed to the north of the Yinshan Mountains and to the west of the Da Hinggan Mountains and was mainly distributed in the northern and central parts of Inner Mongolia (Figure 4a). The frequency of cold surges in the study area from 1960 to 2012 displayed a "bimodal structure" (i.e., the proportion of frequencies between 8 and 10 times and $14-16$ times were $26.17 \%$ and $17.28 \%$, respectively) (inset plot in Figure 4a). We further analyzed spatial characteristics based on eco-zones. Result showed significant
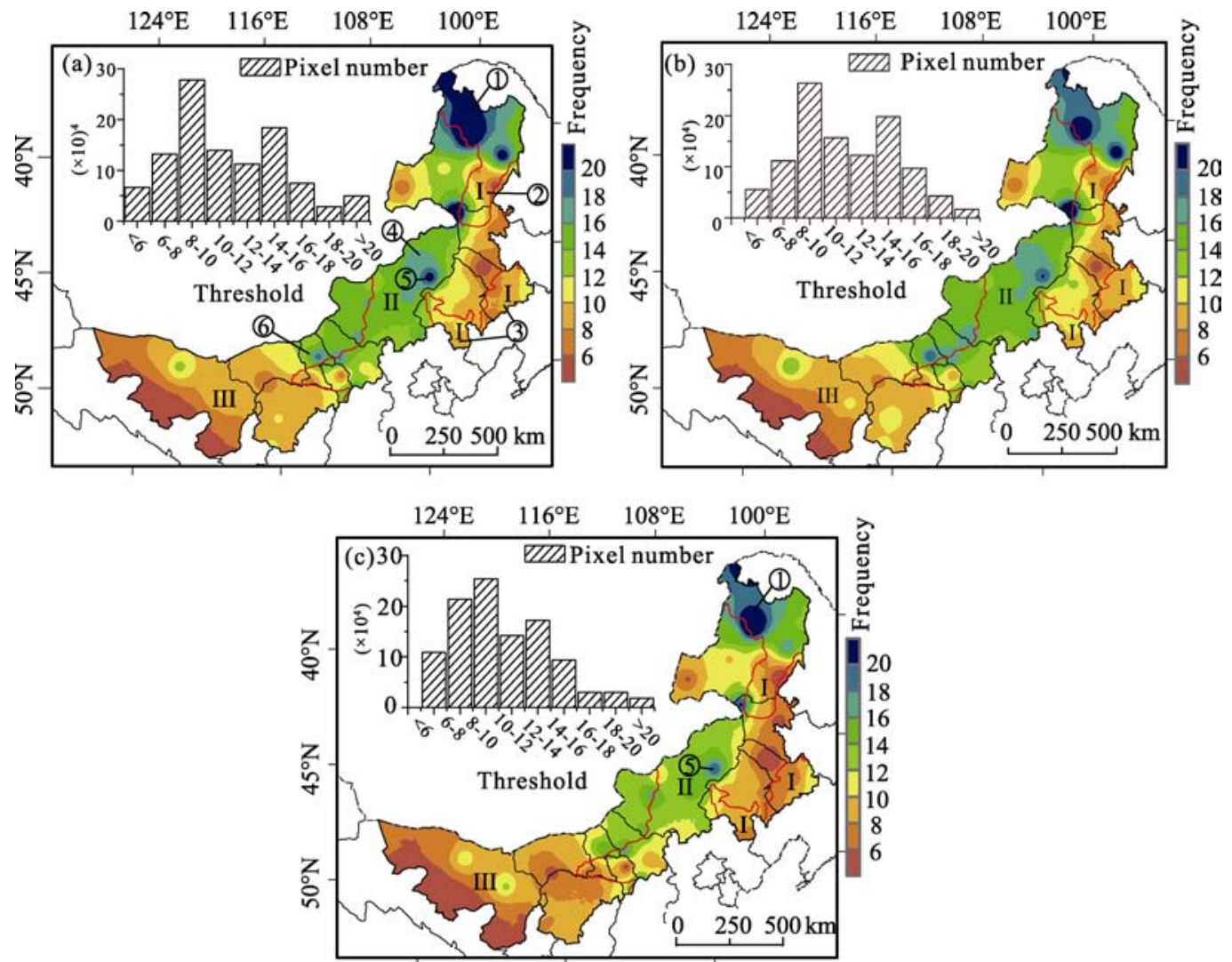

Figure 4 Spatial distribution of cold surge frequency at each station in Inner Mongolia 
spatial differences in forested regions, with more than 20 cold surges occurring north of the Da Hinggan Mountains (1) and less than 10 cold surges occurring in the middle of the Da Hinggan Mountains (2) and the western Liaohe Plain (3). The frequency of cold surges was greater than 14 in grassy regions, and high values were mainly observed in the eastern part of the Inner Mongolia Plateau (4), with the highest value occurring in Xi Ujimqin Banner (5). In desert regions, a decreasing trend in the frequency of cold surges occurred from the northeast to the southwest. The frequency of cold surges in both the western Hetao (Loop) Plain and the western part of the Inner Mongolia Plateau (6) was more than 14 times, while a frequency of less than 10 times was observed to the west of this region.

We further analyzed spatial distribution before and after the 1991 turning point, which was detected by piecewise regression modeling. The spatial pattern of cold surge frequency between 1960 and 1991 was similar to the spatial frequency pattern observed from 1960 to 2012 (Figure 4b). Obvious differences were apparent in regions with a high occurrence of cold surges (more than 20 times), which were generally located north of the Da Hinggan Mountains (1). Prior to 1991, regions with high cold surge frequency covered less area that was observed between 1960 and 2012. This phenomenon illustrates that regional high frequency values were mainly caused by elevated values observed between 1991 and 2012, which is further demonstrated by Figure 4c. In addition, a decreasing trend was observed in grasslands after 1991, and regions with high values were scattered in the Xi Ujimqin Banner (5) and the western Hetao Plain (6) (Figure 4c). On a decadal scale, regions with high cold surge frequency presented a decreasing trend, and regions with low cold surge frequency showed an increasing trend between 1960 and 2000 (not shown). Regions with a high cold surge frequency increased in recent years, further demonstrating the increase of the frequency and intensity of extreme climatic events caused by global climate change. As can be seen from the above analysis, regions with a high occurrence of cold surges were generally located on the boundary of a forested and a grassy region. Furthermore, the frequency and intensity of cold surges increased in recent years. Therefore, the spatial overlay of cold surges and forest and grassy region will inevitably pose a serious threat to local agricultural production.

\subsubsection{Spatial distribution of cold surge trends}

We calculated the Sen Trend of cold surge frequency for the period between 1960 and 2012 and for the periods before and after the turning point (1991), which was detected by piecewise regression. The significance of trends was further tested using the Mann-Kendall statistical test. Figure 5 illustrates that the trends for the entire study period were between -2.4 times/10a and 1.2 times/10a, and areas with significant decreasing trends encompassed 93.04\% of the entire study area. Regions with significant decreasing trends were mainly distributed in the northwestern area of Xingan Meng, the northeastern area of Xilin Gol League, and Baotou. Comparatively, areas with significant increasing trends were distributed in Tulihe (station with black circle in Figure, the same marked in the following text of this section) of Hulun Buir and Bayan Mod of Alxa League.

From 1960 to 1991, cold surge frequency trends were between -3.3 and 2.5 times/10a (Figure 5b), which was more drastic than that observed for the entire study period. More than $99 \%$ of the entire study area presented a decreasing trend during 1960-1991, and areas 
with more than -1 times/10a possessed $70.82 \%$ of the entire study area (significantly more than $12.67 \%$ determined for the entire study period) and were primarily distributed at Chifeng station in Chifeng, Ongniud Banner station in Chifeng, Suolun station in Xingan Banner, Dong Ujimqin Banner station in Xilin Gol League, and Ejin Banner station in Alxa League. Trends from 1991 to 2012 were between -4.4 and 4.2 times/10a (Figure 5c), and areas with an increased cold surge frequency (76.28\%) were larger than areas with decreased cold surge frequency (23.72\%). Areas with significant increasing trends were mainly distributed in Xilin Hot and Xi Ujimqin Banner.

As can be seen from the above analysis, the trends acquired from the entire study period were not explained well by trends in the frequency of cold surges, while the trends obtained from piecewise regression models better characterized the trends in different periods. Accurate analysis of trends in cold surges is vital to comprehensively understand changes in extreme climatic events caused by global climate change.
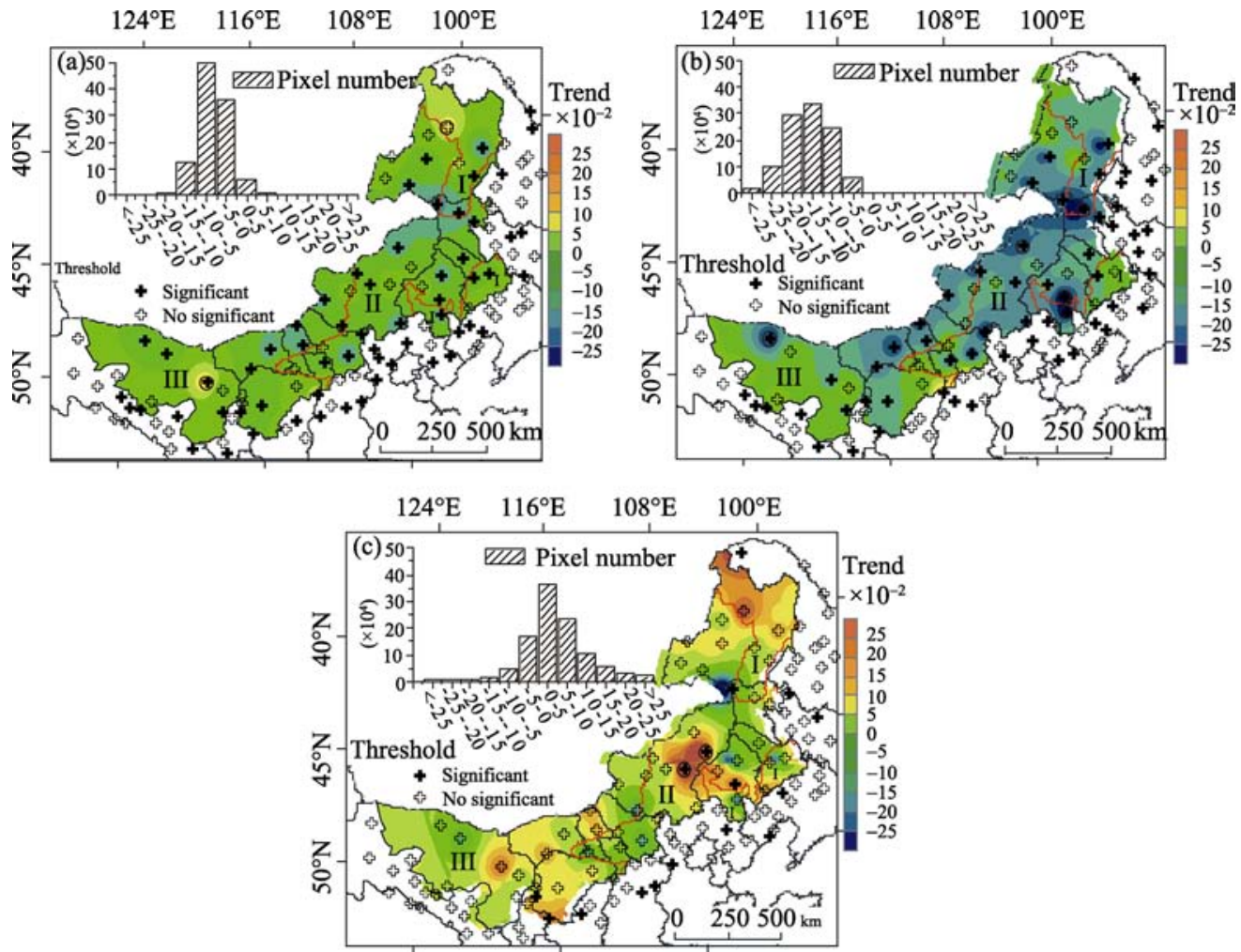

Figure 5 Spatial distribution of cold surge trends in Inner Mongolia from 1960 to 2012

\section{Influencing factors}

\subsection{Atmospheric circulation}

Previous studies have shown that oscillations in climate systems can cause abnormalities in regional atmospheric circulation and, thus, induce characteristic changes in regional climate (Ding et al., 1991; Wen et al., 2000). In order to analyze the possible driving forces of cold 
surges, we calculated correlation coefficients between cold surge frequency and circulation factors using the correlation analysis method (Table 1). We found a significant negative correlation between cold surge frequency and both $\mathrm{AO}$ and $\mathrm{NAO}$, with correlation coefficients of $-0.41(\mathrm{p}<0.01)$ and -0.31 ( $<<0.05)$, respectively. Both the correlation of cold surge frequency with $\mathrm{AO}$ and $\mathrm{NAO}$ in winter months showed a significant negative correlation of $-0.36(\mathrm{p}<0.01)$ and $-0.40(\mathrm{p}<0.01)$, respectively. In contrast, a significant positive correlation was detected between cold surge frequency and both CA and APVII, with correlation coefficients of $0.50(\mathrm{p}<0.01)$ and 0.47 ( $\mathrm{p}<0.01)$, respectively. CA was significantly correlated with cold surges for all seasons, and APVII was significantly correlated with cold surge only during winter and spring.

In addition, cold surge frequency was significantly correlated with TPI, SHI, CW, and IZ in winter and with IM in autumn. On an annual scale, cold surge was primarily correlated with AO, NAO, CA, APVII, and CQ. However, differences in driving forces existed between different seasons. The winter cold surge was significantly correlated with all circulation factors except CQ and IM, which demonstrates that winter cold surges were controlled by multiple forces. While the autumn cold surge was mainly correlated to CA and IM, the spring cold surge was correlated with CA and APVII. In order to better understand the influence of cold surge driving forces, we further analyzed changes in the primary driving factors, which were chosen based on the above analysis.

The Arctic Oscillation (AO) has a significant effect on atmospheric circulation and climatic factors in the Northern Hemisphere (Yao et al., 2014). In winter, the AO affects the intensity of winter monsoons through its influence on regional atmospheric circulation (i.e., changes to the Siberian High and East Asian trough that cause regional temperature changes). In this study, the AO showed an increasing trend from 1960 to 2012. However, a turning point from a negative phase to a positive phase was detected in 1989 using a piecewise regression model, which is consistent with conclusions drawn by Chen Yuying (Chen et al., $2010)$. The AO presented an increasing trend $(p=0.07)$ prior to 1989 , reflecting a gradual weakening of the polar high pressure and a gradual increase of mid-latitude low pressure, which controls cold air in polar regions through a strengthening of the polar vortex. Therefore, the intensity of the Siberian High and winter monsoon decreased, resulting in a reduction of the frequency of cold surges. The analysis above is consistent with the cold surge frequency trend in our study. The AO showed a decreased trend $(p=0.12)$ after 1989 with an increase in the negative phase and a weakening of the polar vortex causing a strengthening of the Siberian High and winter monsoons and, thus, an increase in the frequency of cold surges. Our results further demonstrated this trend. Similar observations were detected in the long-term trend of the North Atlantic Oscillation (NAO) index, which illustrates that AO and NAO were important factors influencing the frequency of cold surges in Inner Mongolia.

Siberian High was a key factor affecting cold surge outbreaks. In our study, the SHI was standardized to mean sea-level pressure, which was calculated between $40^{\circ} \mathrm{N}$ and $60^{\circ} \mathrm{N}$ and between $80^{\circ} \mathrm{E}$ and $120^{\circ} \mathrm{E}$. We found that cold surge frequency displayed a significant positive correlation with SHI during winter, while a weak positive correlation was present in both autumn and spring. This may be attributed to the fact that the SHI was strong in winter and, thus, strongly affected cold surges during winter months. Contrastingly, the intensity of the SHI was relatively weak during autumn and spring, which was consistent with previous 
findings (Wang et al., 2006).

It should be noted that Gong Daoyi established SHI trends for the most recent century (Gong et al., 2003). He showed that the strongest SHI occurred in the 1960s and subsequently weakened during the 1980s and 1990s. These results coincided with decadal change characteristics of cold surges determined in this study, further confirming that the SHI was an important factor influencing cold surge frequency in China. Long-term trends indicate that the 1991 turning point in cold surge frequency was also apparent in the SHI. Prior to 1991, cold surge frequency and SHI trends were consistent. However, after 1991, SHI trends were much higher than those observed for cold surge frequency.

Table 1 Correlation coefficients between cold surge frequency and its influencing factors

\begin{tabular}{ccccccccccc}
\hline Factors & AO & NAO & SHI & CA & TPI & APVII & CQ & CW & IZ & IM \\
\hline Annual & $-0.41^{* *}$ & $-0.31^{*}$ & 0.13 & $0.50^{* *}$ & 0.11 & $0.47^{* *}$ & $-0.34^{*}$ & -0.08 & 0.22 & 0.21 \\
Autumn & -0.13 & 0.05 & 0.15 & $0.33^{*}$ & -0.01 & 0.23 & -0.13 & -0.17 & 0.18 & $0.35^{*}$ \\
Winter & $-0.36^{* *}$ & $-0.40^{* *}$ & $0.32^{*}$ & $0.45^{* *}$ & $0.37^{* *}$ & $0.48^{* *}$ & -0.10 & $-0.32^{*}$ & $0.29^{*}$ & 0.06 \\
Spring & -0.14 & -0.13 & 0.08 & $0.38^{* *}$ & 0.08 & $0.39^{* *}$ & -0.00 & -0.12 & 0.25 & 0.13 \\
\hline
\end{tabular}

Note: $*$ and $* *$ indicate significance at the $\mathrm{p}<0.05$ and $\mathrm{p}<0.01$ levels, respectively.

\subsection{Topography}

Local climate is considered to be a complex combination of atmospheric circulation and geographical conditions. Of these, atmospheric circulation can explain the spatial distribution of cold surges on a large scale, while the internal terrain differences of a given region may strongly influence the uneven distribution of cold surge frequency and intensity. Topography plays a key role in redistributing hydrothermal conditions through the blocking or shunting of cold air. Therefore, the impact of topography on cold surges was significant. Our study area contains two primary mountain ranges: the Da Hinggan Mountains extend from the northeast to the southwest in the eastern part of the study area, and the Yinshan Mountains extend from east to west in the central part of the study area. The angled region formed by the two mountain ranges is directly affected by cold air from Siberia and, thus, the region of Inner Mongolia is most severely affected by cold surges. Comparatively, the frequency of cold surges to the east of the Da Hinggan Mountains and to the south of the Yinshan Mountains was relatively less. Therefore, obvious spatial differences in cold surge frequency occurred in our study area. These differences may have been caused by the cold air surges through the western foot of the Da Hinggan Mountains and the northern foot of the Yinshan Mountains after the cold air outbreaks. On the one hand, the blocking effect of the mountains cause cold air to climb slowly and increase cold air retention causing increased cold surge frequencies in this region. On the other hand, the "easy in and difficult out" (Huang et al., 1986; Lv et al., 1956) terrain features of the region, which are triggered by the two mountain ranges, worsened the damages caused by cold surges. Under the influence of gravity, cold air moves faster after passing over the mountain ranges and, therefore, only affects these local climates for a short time. Thus, cold surge frequency was lower east of the Da Hinggan Mountains and south of the Yinshan Mountains. 


\section{Conclusions and discussion}

\subsection{Conclusions}

Based on daily minimum temperature data from 121 meteorological stations in Inner Mongolia and the surrounding areas and circulation factors, we analyzed the spatiotemporal variation of cold surges in Inner Mongolia between 1960 and 2012 and its driving forces by using piecewise regression model, Sen + Mann-Kendall model, and correlation analysis. Several conclusions can be drawn as follows:

(1) The frequency of single-station cold surges in Inner Mongolia presented a decreasing trend between 1960 and 2012, with a linear tendency of -0.5 times/10a ( -2.4 to 1.2 times/10a). Prior to 1991, a significant decreasing trend of -1.1 times/10a ( -3.3 to 2.5 times/10a) was detected, while an increasing trend of 0.45 times/10a (-4.4 to 4.2 times/10a) was observed after 1991. On a seasonal scale, spring cold surge trends were consistent with annual trends, and the most obvious change in cold surges occurred during spring. The frequency of monthly cold surges displayed a bimodal structure, and there was a high occurrence of cold surges during October, November, and March, with November experiencing the highest incidence of cold surges.

(2) Significant spatial differences were present in cold surge frequency. High incidences of cold surges were mainly observed in the northern and central parts of Inner Mongolia, with higher values observed in northern areas than in central regions. Inter-decadal analyses also revealed that high frequency and low frequency regions experienced decreasing and increasing trends, respectively, between 1960 and 1990. After the 1990s, high frequency regions expanded and were mainly distributed in Tulihe, Xiao'ergou, and Xi Ujimqin Banner. In addition, the overlap of cold surge regions with forested and grassy regions will inevitably pose a serious threat to local agricultural production.

(3) Regions with significant decreasing trends encompassed 93.04\% of the entire study area between 1960 and 2012. Between 1960 and 1991, regions with significant decreasing trends were greater than between 1960 and 2012, with greater than 99\% of the study area presenting a decreasing trend. Of this $99 \%, 70.82 \%$ experienced at least -1 times/10a, which was significantly more than the $12.67 \%$ for the entire study period. Between 1991 and 2012, areas with increased cold surge frequency were larger than areas with decreased frequency (76.28\% and $23.72 \%$, respectively), and areas with significant increasing trends were mainly distributed in Xilin Hot and Xi Ujimqin Banner.

(4) On an annual scale, the cold surge was dominated by AO, NAO, CA, APVII, and CQ, while a seasonal difference in driving forces was detected. Winter cold surges were significantly correlated with AO, NAO, SHI, CA, TPI, APVII, CW, and IZ, indicating that winter cold surges were caused by multiple factors. Autumn cold surges were primarily correlated with CA and IM, while spring cold surges were significantly correlated with CA and APVII. Cold surge frequency presented an increasing trend, which is consistent with the increase in the frequency and intensity of extreme climatic events caused by global climate change.

\subsection{Discussion}

Due to global warming, the characteristics of atmospheric circulation and the frequency and intensity of extreme climatic events have undergone significant changes. Thus, we should 
focus on strengthening our understanding of the spatiotemporal variation of extreme climatic events and the effects of these events on ecosystems. As ecosystems gradually adapt to a warmer climate caused by global warming, the vulnerability of ecosystems to extremely low temperatures will increase. Therefore, once a cold surge occurs, it will cause huge and far-reaching effects on the structure and function of ecosystems.

It should be noted that spring is a critical time for planting crops. If crops experienced cold surges at this stage, huge economic losses would be suffered. Therefore, it is of pivotal importance to increase understanding of spring cold surges to ensure the safety of agricultural production. Previous studies indicated that vegetation coverage significantly decreased in the southeastern part of the Xilin Gol Plateau over the past 10 years (Mu et al., 2012), which is consistent with regions experiencing a high cold surge frequency in our study. However, further research is required to determine whether cold surges, in particular spring cold surges, are one of the factors affecting vegetation coverage in these regions.

Finally, cold surges will also affect the green-up of grasslands, especially with the recent increase of cold surges in grassy regions. Unfortunately, the impact of cold surges on green-up is still unclear. Therefore, in order to enhance our ability to adapt to climate change and strengthen our comprehensive disaster prevention and mitigation capabilities, we should continue to focus on the spatiotemporal variation of cold surges and strengthen research on the risk assessment of cold surges.

\section{References}

Chen Wen, Graf H F, Ronghui H, 2000. The interannual variability of East Asian winter monsoon and its relation to the summer monsoon. Advances in Atmospheric Sciences, 17(1): 48-60.

Chen Yeguo, He Dongyan, Nong Mengsong, 2008. Change of cold wave in the Nanning area under global warming. Advances in Climate Change Research, 4(4): 245-249. (in Chinese)

Chen Yuying, Chen Nan, Ma Jinren et al., 2010. Variety of Ningxia cold waves in the last 48 years and its possible reasons. Journal of Natural Resources, 25(6): 939-951. (in Chinese)

Cui Linli, Shi Jun, Zhou Weidong, 2009. Characteristics of extreme temperature variations and their response to urbanization in Shanghai. Scientia Geograhpica Sinica, 29(1): 93-97. (in Chinese)

Ding Yihui, Wen Shigeng, Li Yunjin, 1991. A study of dynamic structures of the Siberian High in winter. Acta Meteorologica Sinica, 49(4): 430-439. (in Chinese)

Easterling D R, Meehl G A, Parmesan C et al., 2000. Climate extremes: Observations, modeling, and impacts. Science, 289(5487): 2068-2074.

Gong Daoyi, Wang Shaowu, 2003. Influence of Arctic Oscillation on winter climate over China. Acta Geographica Sinica, 58(4): 559-568. (in Chinese)

Guo Linghui, Wu Shaohong, Zhao Dongsheng et al., 2013. Change trends of growing season over Inner Mongolia in the past 50 years. Scientia Geographica Sinica, 33(4): 505-512. (in Chinese)

Huang Shoubo, 1986. Advance in the investigation of the topographical microclimate in China. Geographical Research, 5(2): 90-101. (in Chinese)

John R, Chen J, Lu N et al., 2008. Predicting plant diversity based on remote sensing products in the semi-arid region of Inner Mongolia. Remote Sensing of Environment, 112(5): 2018-2032.

Kendall M G, 1948. Rank Correlation Methods. London: Charles Griffin \& Company Limited.

Li Xianzhi, 1955. The research of cold wave in East Asia. China modern science on the election issue. (in Chinese)

Lv Jiong, 1956. Topographical features and frost damage. Acta Geographica Sinica, 22(2): 149-158. (in Chinese)

Mansur Sabit, 2012. Climatic characteristics of cold wave and its impact on agriculture in southern Xinjiang in 
recent 60 years. Journal of Natural Resources, 27(12): 2145-2152. (in Chinese)

Mu Shaojie, Yang Hongfei, Li Jianlong et al., 2013. Spatiotemporal dynamics of vegetation coverage and its relationship with climate factors in Inner Mongolia, China. Journal of Geographical Sciences, 23(2): 231-246.

National Assessment Report on Climate Compiling Committee (NARCCC), 2011. Second National Assessment Report on Climate Change. Beijing Science Press. (in Chinese)

Qian Weihong, Zhang Weiwei, 2007. Changes in cold wave events and warm winter in China during the last 46 years. Chinese Journal of Atmospheric Sciences, 31(6): 1266-1278. (in Chinese)

Sen P K, 1968. Estimates of the regression coefficient based on Kendall's tau. Journal of the American Statistical Association, 63(324): 1379-1389.

Stocker D Q, 2013. Climate change 2013: The Physical Science Basis. Working Group I Contribution to the Fifth Assessment Report of the Intergovernmental Panel on Climate Change, Summary for Policymakers, IPCC.

Sun Yanling, Guo Peng, Yan Xiaodong et al., 2010. Dynamics of vegetation cover and its relationship with climate change and human activities in Inner Mongolia. Journal of Natural Resources, 25(3): 407-414. (in Chinese)

Sun Jinyu, Wang Xuhui, Chen Anping et al., 2011. NDVI indicated characteristics of vegetation cover change in China's metropolises over the last three decades. Environmental Monitoring and Assessment, 179(1-4): 1-14.

Tao Shiyan, 1957. A synoptic and aero logical study on a cold wave in the Far East during the period of the breakdown of the blocking situation over Euroasia and Atlantic. Acta Meteorologica Sinica, 28(1): 63-74. (in Chinese)

Tomé A R, Miranda P M A, 2004. Piecewise linear fitting and trend changing points of climate parameters. Geophysical Research Letters, 31, L02207.

Wang Mingjiu, Zhang Cunhou, 2013. Climate change in Inner Mongolia grassland and the effects on pastoral animal husbandry. Inner Mongolia Prataculture, 25(1): 5-12. (in Chinese)

Wang Zhengwang, Zhao Guixiang, Zhang Ruiting et al., 2010. Characteristic analysis of cold wave weather in southeast area of Shanxi Province during last 40 years. Journal of Natural Disasters, 19(6): 55-60. (in Chinese)

Wang Zunya, Ding Yihui, 2006. Climate change of the cold wave frequency of China in the last 53 years and the possible reasons. Chinese Journal of Atmospheric Sciences, 30(6): 1068-1076. (in Chinese)

Yao Junqiang, Liu Zhihui, Yang Qing et al., 2014. Temperature variability and its possible causes in the typical basins of the arid Central Asia in recent 130 years. Acta Geographica Sinica, 69(3): 291-302. (in Chinese)

Zhai Panmao, Ren Fumin, 1997. On changes of China's maximum and minimum temperatures in the recent 40 years. Acta Meteorologica Sinica, 55(4): 418-429. (in Chinese)

Zhai Panmao, Sun Anjian, Ren Fumin et al., 1999. Changes of climate extremes in China. Climatic Change, 42(1): 203-218.

Zheng Jingyun, Hao Zhixin, Fang Xiuqi et al., 2014. Changing characteristics of extreme climate events during past 2000 years in China. Progress in Geography, 33(1): 3-12. (in Chinese)

Zhou Yaqing, Ren Guoyu, 2011. Change in extreme temperature event frequency over mainland China, 1961-2008. Climate Research, 50(2): 125-139. 